\title{
Technology for Working Dogs
}

Melody Moore Jackson melody@cc.gatech.edu Ceara Byrne

ceara.byrne@gatech.edu Larry Freil

larry.freil@gatech.edu

Giancarlo Valentin

giancarlo@gatech.edu

Jay Zuerndorfer

jzuerndorfer@gatech.edu

Clint Zeagler

clintzeagler@gatech.edu

Jacob Logas

logasja@gatech.edu

Scott Gilliland

scott.gilliland@gatech.edu
Allison Rapoport

arapoport7@gatech.edu

Shuyi Sun

zeusspade@gatech.edu

Devon Peet

dpeet@gatech.edu

Andrea Lau

andrea.lau@gatech.edu

Xiaochuang Han

xc@gatech.edu

Joelle Alcaidinho

joelle@gatech.edu

Thad Starner

thad@cc.gatech.edu

Georgia Institute of Technology

Atlanta, GA 30306, USA

Permission to make digital or hard copies of part or all of this work for personal or classroom use is granted without fee provided that copies are not made or distributed for profit or commercial advantage and that copies bear this notice and the full citation on the first page. Copyrights for third-party components of this work must be honored. For all other uses, contact the owner/author(s).

Copyright held by the owner/author(s).

ACl18, December 4-6, 2018, Atlanta, GA, USA

ACM 978-1-4503-6219-1/18/12.

https://doi.org/10.1145/3295598.3295615

\begin{abstract}
Working dogs can perform many tasks that improve the

lives of humans. An explosion of new technologies in AnimalComputer Interaction enable dogs to assist in a variety of scenarios. Dogs can summon emergency services, report finding a lost child, determine what explosive they just detected, alert to a tornado siren, and much more. Giving working dogs the ability to clearly communicate could be life-saving. Related technologies can measure aspects of a dog's behavior to assess temperament, predict training success, and even monitor health. The Georgia Tech Animal-Computer Interaction lab has been researching dog-centered interfaces since 2012. Our studies have included instrumented vests, touchscreens, and devices that provide new ways for dogs to communicate what they perceive to people around them. We have also researched instrumented dog toys for temperament analysis and health monitoring. For the ACl2018 demonstrations, we showcase the technologies we have developed for dogs and the new interactions these technologies enable.
\end{abstract}

\section{Author Keywords}

Animal computer interaction; technology; wearable technology; touchscreen; virtual user interface; tangible user interface; physical computing. 


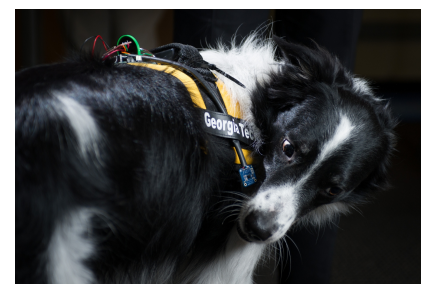

Figure 1: Alerting using the proximity sensor.

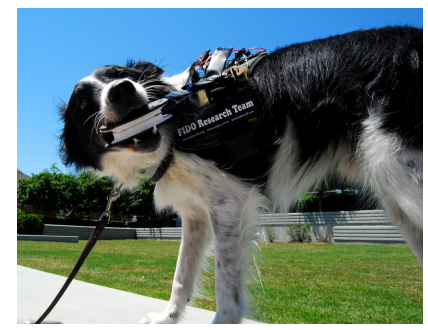

Figure 2: Activating on-body bite sensor.

\section{Introduction}

Working dogs benefit society in many ways: helping individuals with visual, hearing, and motor disabilities; finding people lost in the wilderness; alerting to medical emergencies; and providing critical functions for police and military missions. Peoples' lives depend on the work these dogs perform every day and our research aims to support working dog teams with technology.

First, we aim to facilitate communication between working dogs and people. By effectively facilitating communication, we reduce confusion or errors in interpreting information from a working dog. Secondly, we are interested in technologies that are effective in any environment. Working dogs can be placed in situations ranging from an airconditioned office building to a forest during a downpour. Finally, we want to ensure that our technology is unobtrusive, intuitive, and comfortable for the dog to use. We try to design our technology in a way that takes the dog's experience in account to the best of our ability.

To accomplish these goals, we developed a variety of scenariodriven devices and systems. We based our interaction technologies on a dog's natural perceptual and cognitive capabilities. We designed sensors that detect natural dog interactions such as biting, chewing, tugging, or nosing. By designing around natural behaviors, we can more easily train dogs to use these devices and systems.

\section{Wearable Dog-Computer Interfaces}

Our lab's initial research focused on designing sensors for service dog interactions. We developed a variety of sensors to measure bites, touches, and tugs allowing a dog to send an unambiguous signal to a person.

\section{Medical Alert}

Our original studies examined basic properties of interfaces for dogs, and we developed and tested nine different types of sensors, including bite sensors (pressure, pneumatic, and capacitive), tug sensors, and touch (proximity) sensors [5]. We incorporated the best sensors into medical alert vests and trained dogs to activate the sensors to summon emergency services or text family members with a GPS location during a medical crisis such as a seizure.

\section{Discrimination Task}

The ability for working dogs to discriminate between objects or substances can play a major role in protecting humans. For example, a bomb detection dog's ability to distinguish between a stable explosive and an unstable explosive could increase the safety of first responders and civilians if the dogs were able to effectively communicate it. A dog's olfactory system is powerful, but often the dogs cannot relay exact details of what they have found to their handler even though they are aware of the difference. Our goal when investigating on-body affordances on working dog vests in 2013 and 2015, as shown in Figure 1 [4, 5], was to use technology to enhance a dog's communicative ability.

The working dog vest was outfitted with a touch sensor on either side and enabled the dog to either touch a sensor on the left or right to communicate. To test this interaction, we trained a dog to recognize the difference between a tornado siren and a fire alarm, and activate the sensor associated with the sound. Another dog was trained to recognize a frisbee or a ball and activate the sensor associated with the object presented, demonstrating that dogs can perform a discrimination task and communicate what they have heard or seen. 


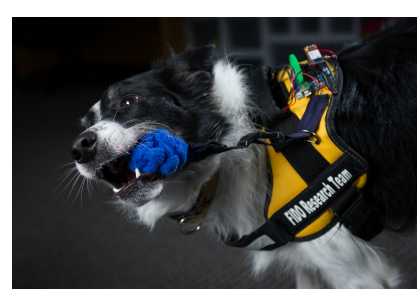

Figure 3: Alerting using tug sensor.
Search and Rescue

[8] An Autistic boy went missing in the mountains of North Georgia in 2013. Search and rescue dog teams were dispatched, and after hours of searching, a dog working at a distance from her handler found the child. Dogs typically perform a "recall and refind", returning to their handlers and then leading the handler to the lost person. However, in this case the child was terrified of dogs and once the dog found him, he ran away. This forced the dog to start a new search each time she found the child and returned to her handler. Our goal was to enhance search dog capabilities and allow them to work more efficiently for more effective search and rescue.

For this scenario, we designed a capacitive touch sensor attached securely to the dog's vest to avoid snagging on environmental objects or interfering with the dog's movements. The electronics were enclosed in waterproof cases, allowing the dog to travel through small streams and ponds as is common when searching. A handheld tracking system allowed the handler to see the dog's path, and to see when and where the dog alerted, allowing the dog to stay with the found person rather than recalling to the handler.

\section{Touchscreen Interfaces for Dogs}

$[9,10]$ The rise of tools for helping people "age in place" has allowed for greater autonomy of the older population. Seniors often adopt pets to help them feel safer in their home, to reduce depression, or for other reasons. Consider the following situation, an elderly woman has suffered a major fall and is unconscious. Her companion dog tries to rouse her but is unable to. The dog approaches the touchscreen and touches a specific series of icons, at which point the emergency operator is alerted by the system of the woman's current condition along with a location and an image of the scene. It also sends an alert and an image to an emergency contact's mobile phone. Now multiple avenues of rescue are on the way.

We designed and built a touchscreen interface for service dogs to relay emergency information from a home or office setting. We conducted studies to measure the ability of dogs to interact with virtual objects and to determine the optimal designs for interfaces. We also performed a longitudinal in-home field study with three dogs trained to alert on a "get help" cue, to demonstrate feasibility.

\section{Instrumented Smart Dog Toys}

Organizations who produce working dogs are having difficulty keeping up with demand. Waiting times can be up to five years. Many police agencies cannot afford to purchase $\mathrm{K} 9$ officers. The is because breeding, raising, and training a working dog takes substantial resources and time, often taking over two years and costing upwards of $\$ 50,000$ per dog [1]. The stringent requirements for a dog that must be able to operate in any environment cause a failure rate of $50-60 \%$ or more. Dogs are typically found unsuitable because of specific personality traits, known as "temperament". Precious resources are wasted on training dogs not temperamentally destined to succeed. However, dogs unsuitable for one working role might be superb in a different one. For example, dogs released from a service dog program for excessive energy can be successful drug detection or medical alert dogs. Those not energetic enough for service work are often better suited for therapy or emotional support. Assessing a dog's temperament early in training allows resources to be allocated efficiently to provide the ideal working role for that dog. In developing sensors for communication, we noted interesting patterns of activation that seemed to correlate to our test dogs' temperaments, and this inspired the idea of instrumenting dog toys to gather data to quantify temperament. We performed 


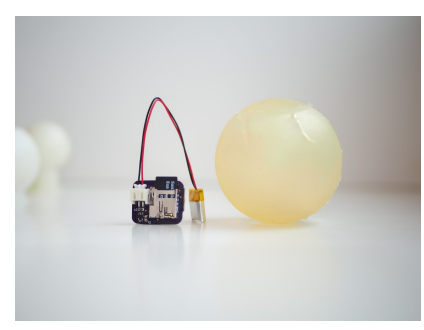

Figure 4: The instrumented ball toy and ball board.

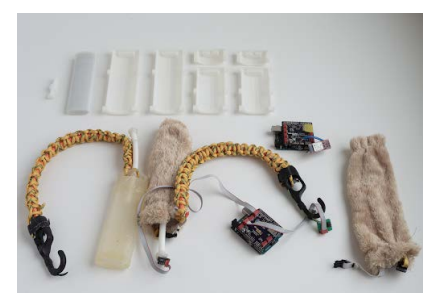

Figure 5: The instrumented tug toys

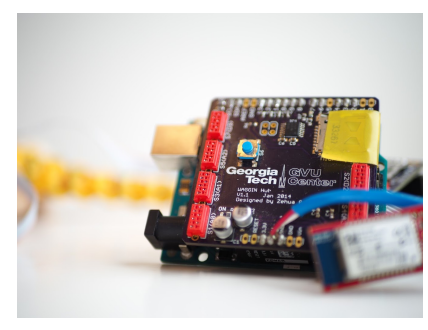

Figure 6: The instrumented tug board a two-year study at Canine Companions for Independence, and found that by testing dogs with the instrumented toys early in the training process, we were able to predict with upwards of $87 \%$ which dogs would succeed or fail.

Another benefit of quantified temperament analysis is for assessment and early intervention, allowing identification and mitigation of behavioral problems which can lead to a dog's failure. Monitoring throughout the training process with instrumented toys may also provide essential information about the dog's progress and health. Slight changes in behavior detected from Smart Toy data may aid in identifying health issues before they are apparent.

\section{Instrumented Ball Toy}

[2] The first toy we developed measured fetch and chase drive. We designed and built a tennis-ball sized toy that could roll freely, allowing the dog to chase. Its size was large enough that it was difficult to swallow, but easy to chew and carry. We made it out of a food-safe silicone rubber which allowed us to place a pressure sensor, accelerometer, and gyroscope inside the toy to measure bite force, frequency, and head-shaking behaviors.

\section{Instrumented Tug Toy}

The tug toy was designed based on another natural dog interaction, biting and tugging on objects. The tug toy was made with a hollow silicone rubber bladder containing a pressure sensor. The bladder was attached to a rubber bungee cord with a stretch sensor. This allowed us to measure pressure and pull force of the dog on the sensor. We also developed different covers for the rubber bladder to see if they affected how the dogs interacted with the sensor.

Instrumented Puzzle Toys

To take the Smart Toys project further, we began studying puzzles and problem solving. This project aims to utilize a ball toy with enclosed puzzle mechanisms to detect actions and choices made by dogs during the engagement. Treats are placed inside puzzle mechanisms within a clear, ventilated ball with two round openings on both ends. The dog demonstrates problem solving by discovering ways to obtain the treat from inside these instruments. We hope to identify salient temperament traits from dogs' interactions with the ball. We hope to measure cognitive ability, tenacity, and frustration tolerance.

\section{Detecting Context in Dog Vocalizations}

Recently, researchers studying canid vocalizations argue that dog vocalizations are meaningful and generalizable in respect to the dog's physical size [7, 3, 6]. Our work uses insights from these previous works and machine learning to classify dog vocalizations in every-day contexts. This study is creating a toolset that could be applicable for other animal vocalizations and could effect the training and deployment of service and support dogs. Service dogs or Search and Rescue dogs can naturally react to stimuli, and the system will decode the meaning which is relayed to the handler.

\section{Discussion}

We have developed a variety of scenario driven devices and technologies for working dogs. These are just examples of the work we are performing as part of the Animal Computer Interaction lab at Georgia Tech. While all of these technologies were developed with specific use cases in mind, such as toys for measuring service dog suitability, or sensors and vest for a search and rescue dog, many of these interaction technologies could be used in a wide variety of applications to benefit the relationship between working dogs and the people they serve. 


\section{REFERENCES}

1. Canine Companions for Independence - FAQs. http:// www.cci.org/about/faqs.html\#question29

2. Ceara Byrne, Jay Zuerndorfer, Larry Freil, Xiaochuang Han, Andrew Sirolly, Scott Gilliland, Thad Starner, and Melody Jackson. 2017. Predicting the Suitability of Service Animals Using Instrumented Dog Toys. Proc. ACM Interact. Mob. Wearable Ubiquitous Technol. Article 1, 4 (2017). DOI :

http://dx.doi.org/10.1145/3161184

3. Tamas Faragó, Peter Pongrácz, Friedrike Range, Zsofia Virányi, and Ãdam Miklosi 2010. 'The bone is mine': affective and referential aspects of dog growls. Animal Behaviour 79, 4 (2010), 917-925. DOI : http: // dx.doi.org/10.1016/j. anbehav. 2010.01.005

4. Melody Moore Jackson, Yash Kshirsagar, Thad Starner, Clint Zeagler, Giancarlo Valentin, Alex Martin, Vincent Martin, Adil Delawalla, Wendy Blount, Sarah Eiring, and Ryan Hollis. 2013. FIDO - facilitating interactions for dogs with occupations. In Proceedings of the 17th annual international symposium on International symposium on wearable computers -ISWC '13. ACM Press, New York, New York, USA, 81. DOI : http:// dx.doi .org/10.1145/2493988.2494334

5. Melody M. Jackson, Giancarlo Valentin, Larry Freil, Lily Burkeen, Clint Zeagler, Scott Gilliland, Barbara Currier, and Thad Starner. 2015. FIDO: Facilitating interactions for dogs with occupations: wearable communication interfaces for working dogs. Personal and Ubiquitous Computing 19, 1 (1 2015), 155-173.
DOI : http://dx.doi.org/10.1007/s00779-014-0817-9

6. T Riede and T Fitch. 1999. Vocal tract length and acoustics of vocalization in the domestic dog (Canis familiaris). The Journal of experimental biology 202 (1999), 2859-2867.

7. Sophia Yin and Brenda Mccowan. Barking in domestic dogs: context specificity and individual identification. DOI

http://dx.doi.org/10.1016/j.anbehav.2003.07.016

8. C. Zeagler, C. Byrne, G. Valentin, L. Freil, E. Kidder, J. Crouch, T. Starner, and M.M. Jackson. 2016. Search and rescue: Dog and handler collaboration through wearable and mobile interfaces. In ACM International Conference Proceeding Series, Vol. 15-17-Nove. DOI : http://dx.doi.org/10.1145/2995257.2995390

9. Clint Zeagler, Scott Gilliland, Larry Freil, Thad Starner, and Melody Jackson. 2014. Going to the dogs. In Proceedings of the 27th annual ACM symposium on User interface software and technology - UIST'14. ACM Press, New York, New York, USA, 497-507. DOI : http://dx.doi.org/10.1145/2642918.2647364

10. Clint Zeagler, Jay Zuerndorfer, Andrea Lau, Larry Freil, Scott Gilliland, Thad Starner, and Melody Moore Jackson. 2016. Canine computer interaction. In Proceedings of the Third International Conference on Animal-Computer Interaction - ACl '16. ACM Press, New York, New York, USA, 1-5. DOI : http://dx.doi.org/10.1145/2995257.2995384 